\title{
Advances in Technology Has Made Critical Care Impersonal
}

\author{
Yatin Mehta ${ }^{1}$ Chitra Mehta ${ }^{1}$ \\ ${ }^{1}$ Medanta Institute of Critical Care and Anesthesiology, Medanta \\ The Medicity, Gurgaon, Haryana, India
}

J Card Crit Care TSS 2018;2:54-56

Recent advancements in technology have revolutionized medical care. Starting from electronic medical records to introduction of sophisticated robotic techniques, all have contributed immensely in transforming patient care worldwide. Automated hospital systems have far-reaching ability leading to improved quality of patient care. ${ }^{1}$ Thanks to digitalization, physicians can now visualize and raise orders for their patients while sitting at home. Radiologic advancements have made understanding of different organ systems simpler in a way that was not possible earlier. Technology has impercolated every aspect and branch of medicine. Medicine is now truely a blend of art and science.

It is estimated that on an average, a company has to shell out U.S. $\$ 2.6$ billion to bring out a drug to the market, right from its inception, phase trials, and final launch. It can be extrapolated to medical devices also that are routinely used for critically sick patients. This investment stands justified keeping patient's welfare and outcome in mind. ${ }^{2}$

Apart from this, technological advancements have not only helped medical practitioners in performing better but have also helped patients get information about their disease easily. There is a rider though to this convenience. Technology has proved to be a double-edged sword. It has been observed to create a void between the patient and treating physician. Whether it is secondary to patients getting insufficient communication, or less of empathy from the physician, or due to having a know-it-all attitude after procuring information from the internet remains a matter of debate. If technology has made life smoother for physicians, it has also made things taxing for them. ${ }^{3}$

Similarly, technology has played a great role in shaping the current critical care. Improvement of technology in therapeutics (e.g., from bulky iron lung to smaller, portable, user friendly ventilators with graphic display, etc.) and monitoring have led to conceptualization of critical care as a separate specialty. In the earlier days, only 3 to $5 \%$ of hospital beds used to be dedicated to intensive care. These days we see approximately $30 \%$ of hospital beds allocated

DOI https://doi.org/

10.1055/s-0039-1684878

ISSN 2457-0206.
Address for correspondence Yatin Mehta, MD, MNAMS, FRCA, FAMS, FIACTA, FICCM, FTEE, Medanta Institute of Critical Care and Anesthesiology, Medanta The Medicity, Sector-38, Gurgaon 12001, Haryana, India (e-mail: yatinmehta@hotmail.com).

to critical care. Critical care has been redefined as a specialty, with advancements in technology. Digitalization has helped intensivists to keep up with dynamics of the challenging environment of an intensive care unit (ICU). Bedside technology such as monitors, ventilators, and drug delivery systems with their alarm systems help in keeping a close watch on changing and abnormal clinical values of patients. Use of ultrasound and echocardiography has sort of metamorphosed bedside critical care.

The downside of technology is that it has resulted in "mechanization" of physicians, in general, as well as in intensive care medical practice. Patients are increasingly being regarded as mechanical objects, made up of interacting systems such as monitor, laboratory, or imaging reports. Somewhere during the progress, the caring human touch has been lost. ICU by its very nature is an impersonal and invasive setting for patients. ${ }^{4}$ Many patients recall their experience of ICU as nightmarish, with no privacy, inadequate sleep due to frequent alarming, and separation from loved ones. To make things worse, rampant mechanization of physicians robs them of their identity as well. Various authors have reported all these factors linked to delay in physical and psychological recovery of patients. ${ }^{5}$

Perhaps this "robotization" process was inevitable. Or perhaps this detachment is a natural defense mechanism of physicians to efficiently treat critically sick patients without getting emotionally drained. This process may also be reducing health care workers' feeling of burnout. All this has led to loss of empathy and human connection which patients need desperately. This, in a way, has also contributed to fading away of the sacred patient-physician bond of earlier days.

Apart from depersonalizing the patient-physician relationship, medical technology has crippled our mental faculty in making a diagnosis on clinical grounds. There is over-reliance on hosts of tests-something that has been termed as "technological tenesmus." There is a tendency to jump to investigations right after a brief history. This habit has flourished not due to a matter of convenience alone, fear of litigation in current times has also prompted in ordering

Copyright @2018 Official Publication of The Simulation Society (TSS), accredited by International Society of Cardiovascular Ultrasound (ISCU).

\section{License terms}

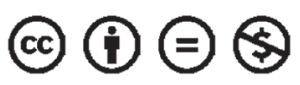


of extensive investigations. In addition, there is no denying that the ritual of physical examination, a must in assessing serious patients, is fast becoming extinct. Nothing can replace a physical touch in practice of medicine. It not only aids in diagnosis but comforts the patient, reinforcing the very fragile patient-physician bonding. ${ }^{6}$

I remember my earlier days of residency when junior physicians and trainees used to religiously record various ventilatory parameters hourly. It was accompanied by a quick clinical look at the patient, helping to pick up warning signs early. Nowadays, monitors, their graphic display, and alarm systems do the job. It has decreased the workload but at the cost of distancing us from our patients.

I still vividly remember an incident where a famous cardiologist brought his mother to the emergency of our hospital. She was an elderly woman with long-standing history of diabetes and hypertension. She presented with left-sided pain in the lower chest of few hours duration. She also had nausea, vomiting, and abdominal discomfort for previous 2 days. She was hemodynamically stable, and electrocardiogram was normal. She did not get any relief with nitrates. She was wheeled into the cardiac cath laboratory within 30 minutes of presentation without a proper examination and cardiac enzymes report. Her coronary angiogram showed normal coronaries. Further workup revealed left hypochondriac tenderness, elevated white cell counts, and increased pancreatic enzymes. Ultrasound of the abdomen confirmed acute pancreatitis.

On more than one occasion, each of us has seen patients who undergo a "million-dollar-workup" with sophisticated technologies without having had the benefit of a detailed history and physical examination. "Technological tenesmus" is thus encouraging hyposkillia among medical practitioners. This hyposkillia may kill medical profession if we continue to train our residents without stressing upon them the importance of history taking and physical eaxmination. ${ }^{7}$ These are essential to develop reasoning power, understand natural history of diseases, and lay out a sound management plan; there is no shortcut. There is no better time than now to endorse these golden words that "patients are our best teachers."

Importance of an early, regular, and effective communication with a patient's family cannot be undermined. It is very essential to hear the family's concerns and make them understand the patient's disease and treatment plan. It helps them in decision making about pertinent goals of treatment. Similar care needs to be taken at the time of patient's death in an ICU. Several institutes have innovated programs to ease out dying patient's and the family's pain, and are trying to make the dying process more humane.

Organ donation is the only chance of survival for patients with end-stage organ failure. It is the only way forward in giving these patients a second chance at life. Organ donation refusal from kin of potential donors restricts organ availability. Effective, empathic, and personalized communication by attending physician goes a long way toward achieving this goal-a "genuine kindhearted" approach. ${ }^{8}$

It is very essential for us to keep health care personalized and people centric. Technology should be used to enhance real life only. Humanism and effectiveness should be discreetly balanced while using high-tech gadgetry. There should be an open face-to-face communication rather than a digitalized connection between physicians and patients. We need to become masters of the technology and not its slaves. Achieving the human side of medicine is not impossible as long as the focus remains on our patient as a human being. There seems to be a serious need to recapture the soul of medicine.

\section{Conflict of Interest}

None.

\section{References}

1 Morrison C, Jones M, Blackwell A, Vuylsteke A. Electronic patient record use during ward rounds: a qualitative study of interaction between medical staff. Crit Care 2008;12(6):R148

2 Lexchin J. Pharmaceutical company spending on research and development and promotion in Canada, 2013-2016: a cohort analysis. J Pharm Policy Pract 2018;11:5

3 Glynn T. The future of the doctor-patient relationship-a critique of current thinking. Health Care and Informatics Review Online 2014;18(1):2

4 McCord G, Pendleton BF, Schrop SL, Weiss L, Stockton L, Hamrich LM. Assessing the impact on patient-physician interaction when physicians use personal digital assistants: a Northeastern Ohio Network (NEON) study. J Am Board Fam Med 2009;22(4):353-359

5 Topçu S, Alper SE, Gülseven B, Kebapçı A. Patient experiences in intensive care units: a systematic review. Patient Exp J 2017;4(3):17

6 Fred HL. The downside of medical progress: the mourning of a medical dinosaur. Tex Heart Inst J 2009;36(1):4-7

7 Fred HL. Hyposkillia: deficiency of clinical skills. Tex Heart Inst J 2005;32(3):255-257

8 Baumgartner F. Human medicine versus techno-medicine. Tex Heart Inst J 2009;36(3):268-269 
